\title{
A GABAergic Inhibitory Component Within the Hippocampal Commissural Pathway
}

\author{
Charles E. Ribak, László Seress, ${ }^{1}$ Gary M. Peterson, ${ }^{2}$ Kim B. Seroogy, ${ }^{3}$ James H. Fallon, and \\ Laurence C. Schmued
}

Department of Anatomy and Neurobiology, University of California at Irvine, Irvine, California 92717

\begin{abstract}
Previous results from immunocytochemical and retrograde transport studies indicated that some GABAergic neurons in the hilus of the dentate gyrus may have axonal projections through the hippocampal commissure. This study has utilized a combined immunocytochemical and retrograde transport method as well as 2 anterograde electron-microscopic methods to determine the existence of this projection in rats. Combined tracer and immunofluorescence studies showed several double-labeled GABAergic neurons in the hilus contralateral to the injection site. The electron-microscopic studies revealed both degenerating and HRP-labeled commissural axons that formed symmetric synapses, the type shown to be formed by GABAergic terminals in the hippocampus. These data demonstrate a GABAergic component within the hippocampal commissural pathway and add further evidence that cortical GABAergic terminals are not derived exclusively from intrinsic neurons.
\end{abstract}

Previous immunocytochemical results have indicated that neurons containing glutamate decarboxylase (GAD) are found throughout the hippocampal dentate gyrus (Ribak et al., 1978). GAD is the synthesizing enzyme for the neurotransmitter GABA, which is known to have an inhibitory effect in the neocortex and hippocampal allocortex (Alger and Nicoll, 1982; Curtis et al., 1970; Krnjević, 1974). A more recent study has shown that the hilar GAD-positive neurons display a wide variety of shapes and sizes (Seress and Ribak, 1983). In addition, a quantitative assessment of these neurons indicated that at least $60 \%$ of the total number of hilar neurons are GABAergic. These data, taken

\footnotetext{
Received Dec. 23, 1985; revised May 8, 1986; accepted May 15, 1986.

The authors gratefully acknowledge Dr. Wolfgang $H$. Oertel for providing the antiserum to GAD, Margot Brundage and Yashoda Jhurani for photographic assistance, John Neff for technical assistance, and Natalie Sepion for secretarial assistance. This work was supported by NIH Grants NS20228 (C.E.R.) and NS 15321 (J.H.F.) and PHS predoctoral fellowships MH 09090 (K.B.S.) and $\mathbf{M H}$ 09121 (L.C.S.).

Correspondence should be addressed to Dr. Charles E. Ribak at the above address.

' Present address: Department of Physiology, University Medical School, Pécs, Hungary.

2 Present address: Department of Neuroscience, University of California at San Diego, La Jolla, CA 92093.

${ }^{3}$ Present address: Department of Histology, Karolinska Institute, S-104 01 Stockholm, Sweden.

Copyright (c) 1986 Society for Neuroscience $0270-6474 / 86 / 123492-07 \$ 02.00 / 0$
}

together with the results of retrograde transport studies that have shown that $80 \%$ of hilar neurons give rise to associational and commissural projections (Berger et al., 1981; Laurberg, 1979; Swanson et al., 1978, 1981; Voneida et al., 1981; West et al., 1979), indicate that many GABAergic hilar neurons are projection neurons.

The notion that some of the GABAergic hilar neurons project to the contralateral dentate gyrus is further supported by our electron-microscopic data on neurons that give rise to the commissural pathway. Utilizing the retrograde transport of HRP, Seroogy et al. (1983) demonstrated the ultrastructural features of hilar commissural neurons and showed 2 populations of labeled somata. One type of soma was similar to that of spiny CA3 pyramidal projection neurons, whereas the other type of labeled commissural soma had ultrastructural features described previously for GABAergic, local circuit neurons (Ribak et al., 1978). Therefore, the data for both the numbers of GABAergic hilar neurons and the ultrastructural features of hilar commissural neurons suggest that some GABAergic hilar neurons have axons that project through the hippocampal commissural pathway.

In the present study, we have used a combination of anatomical methods to show that GABAergic hilar neurons have commissural projections. First, a combined GAD immunocytochemical and fluorescence retrograde-tracing method was used to directly demonstrate hilar neurons that are GABAergic and have commissural projections. In addition, anterograde degeneration and anterograde transport of HRP methods were used to show that the commissural pathway gives rise to some axon terminals that form symmetric synapses, the type that has been shown to be GABAergic in the hippocampus (Kosaka et al., 1984; Ribak et al., 1978; Somogyi et al., 1983). The data from these studies indicate that the hippocampal commissural pathway has a GABAergic inhibitory component. Some of these data have been previously presented in abstract form (Ribak et al., 1985).

\section{Materials and Methods \\ Combined retrograde tracing and immunocytochemistry}

Twenty adult Sprague-Dawley rats of both sexes (250-300 gm) were used in this sct of experiments. With animals under sodium pentobarbital anesthesia, $100 \mathrm{nl}$ of Fast blue $\left(7 \%\right.$ in distilled $\left.\mathrm{H}_{2} \mathrm{O}\right), 50 \mathrm{nl}$ of Fluoro-

Figures 1-6. Fluorescence photomicrographs contralateral to injection sites of tracers to show examples of double-labeled GABAergic neurons (arrows) in the hippocampal formation. Figure 1 shows numerous somata that are retrogradely labeled with Fluoro-Gold in the hilus. Two of these somata (arrows) also contain immunofluorescence for GAD in Figure 2. Note that the granule cell layer (G) lacks somal labeling. Figure 2 shows GAD immunofluorescence in the dentate gyrus. Two GAD-positive somata (arrows) are double-labeled (cf. Fig. 1). One soma lies below the granule cell layer $(G)$, which contains the pericellular GAD-positive basket plexus, whereas the other soma is deeper in the hilus. The same blood vessels are indicated by asterisks. Figures 3 and 4 show 2 more examples of retrogradely labeled (Fig. 3) GAD-positive (Fig. 4) commissural neurons. Figures 5 and 6 show an example of a retrogradely labeled (Fig. 5) GAD-positive (Fig. 6) nonpyramidal cell in the CA3 pyramidal cell layer (P). $\times 340$. 

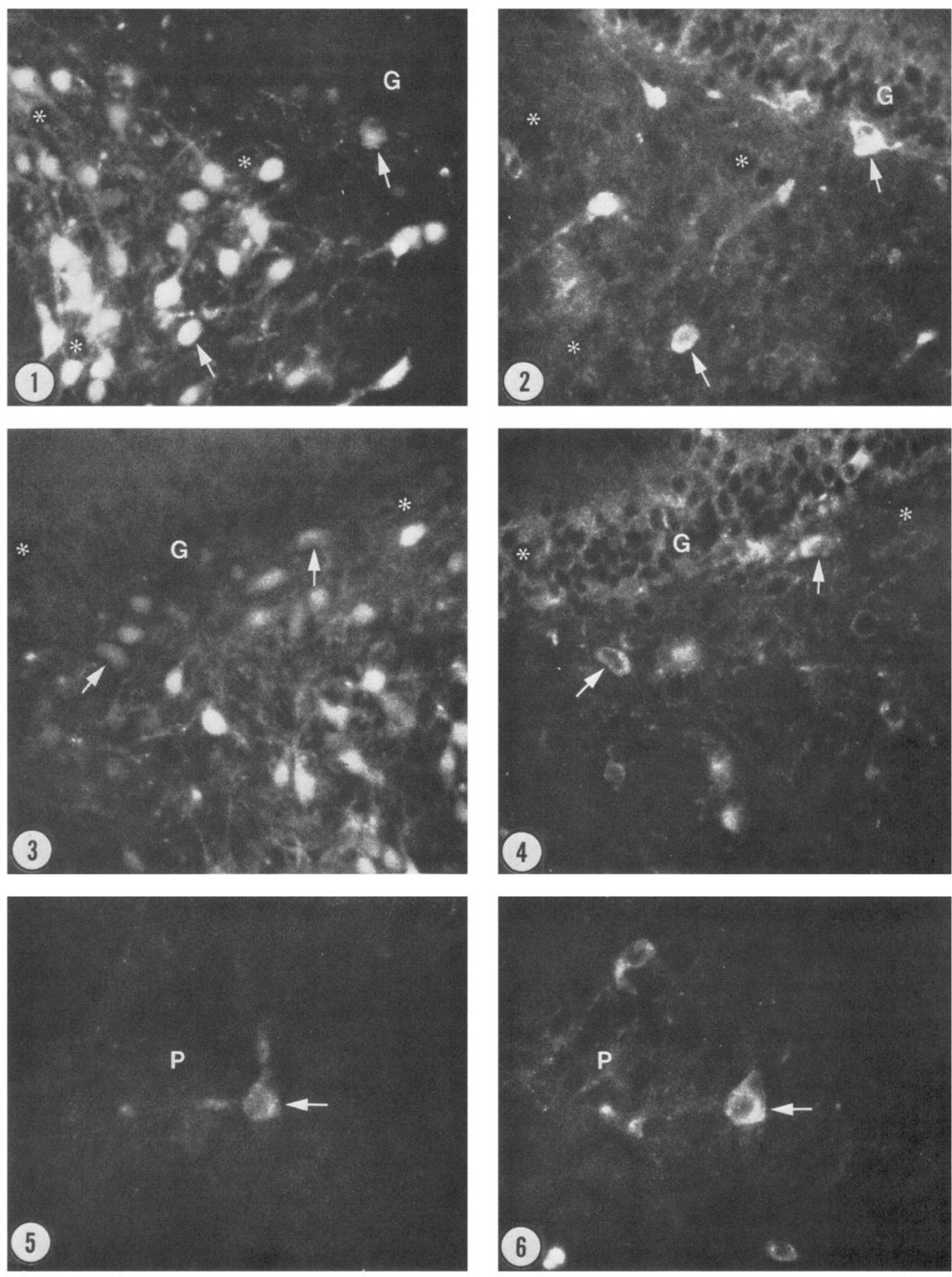

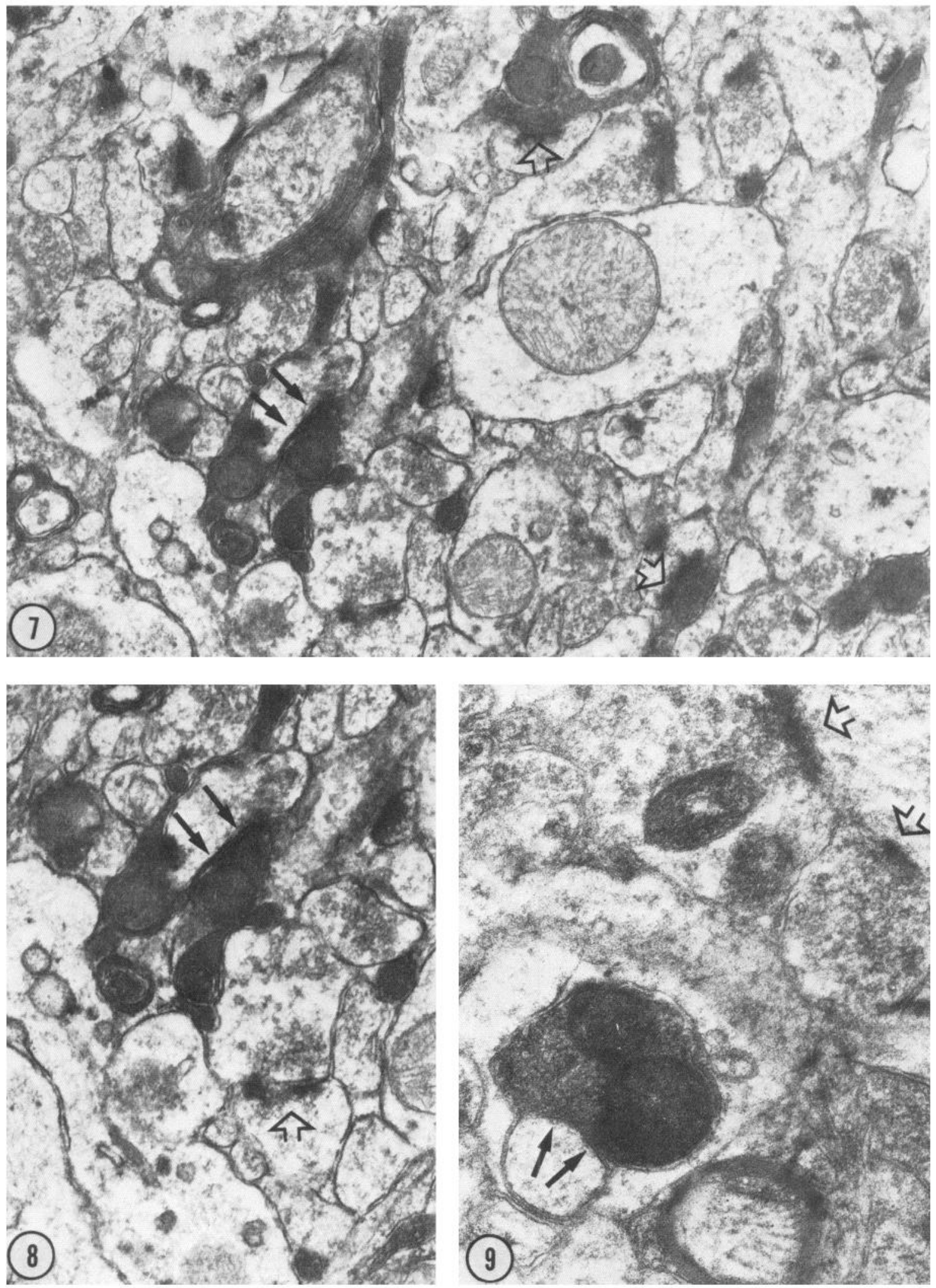

Figures 7-9. Electron micrographs of degenerating commissural axons in the stratum oriens of the CA1 dorsal hippocampus. Figure 7 shows numerous profiles of degenerating axons. One degenerating terminal (open arrow) forms an asymmetric synapse with a spine, whereas another terminal (arrows) forms a symmetric synapse. $\times 13,000$. Figure 8 shows an enlargement of this latter synapse (arrows) and an adjacent nondegenerating terminal that forms an asymmetric contact (open arrow) $\times 20,000$. Figure 9 shows another example of a degenerating commissural terminal that forms a symmetric synapse (arrows) with a small spine. Two nondegenerating terminals form asymmetric synapses (open arrows) with a dendritic shaft. $\times 40,000$. 
Gold ( $2 \%$ in saline; Fluorochrome, Englewood, CO) (Schmued and Fallon, 1986), or $100 \mathrm{nl}$ of WGA-HRP (10\%) was stereotaxically injected into the dentate gyrus. The injections were completed over a $10 \mathrm{~min}$ time period, after which the tip of the syringe was allowed to remain in place for an additional $10 \mathrm{~min}$ before withdrawal from the brain. One or $3 \mathrm{~d}$ following the dye injections, rats were injected intraventricularly with $70-100 \mu \mathrm{g}$ colchicine $(10 \mu \mathrm{g} / \mu \mathrm{l})$, and perfused $24-48 \mathrm{hr}$ later. Animals that received WGA-HRP injections were injected with colchicine $1 \mathrm{~d}$ later and then perfused the following day. In brief, the animals were perfused transcardially with room-temperature $0.1 \mathrm{M}$ PBS ( $\mathrm{pH} \mathrm{7.4)} \mathrm{for} 5 \mathrm{~min}$, followed by cold 4\% paraformaldehyde in PBS for $15 \mathrm{~min}$. The brains were immediately removed from the cranium and placed in a solution of the above fixative with $20 \%$ sucrose, and refrigerated overnight at $4^{\circ} \mathrm{C}$.

Frozen sections were cut in the coronal plane at a thickness of 10 or $40 \mu \mathrm{m}$ with a cryostat or sliding microtome, respectively. The thinner sections were processed for immunocytochemistry after mounting onto gelatin-coated slides, whereas the thicker sections were processed as free-floating sections and then mounted. The sections of those brains that had received injections of fluorescent dye were processed using a modification of the indirect immunofluorescence procedure of Coons (1958). After two $10 \mathrm{~min}$ rinses in PBS, the sections were incubated for $\mathrm{l} \mathrm{hr}$ at room temperature and overnight at $4^{\circ} \mathrm{C}$ in "blocking medium," consisting of $10 \%$ normal rabbit serum (NRS) and $0.1 \mathrm{M} \mathrm{D,L-lysine} \mathrm{in}$ $0.05 \mathrm{M}$ Tris-buffered saline (TBS) to reduce nonspecific staining of tissue. Antiserum to GAD (Oertel et al., 1981) was applied at a dilution of 1:500 in TBS, followed by overnight incubation at $4^{\circ} \mathrm{C}$. After several 10 min washes in TBS, the sections were incubated with FITC-conjugated rabbit anti-sheep IgG at a 1:100 dilution in TBS for $1 \mathrm{hr}$ at room temperature. Following three $5 \mathrm{~min}$ rinses in TBS, the sections were mounted, dried, coverslipped with a $6: 1$ solution of glycerine-TBS, and examined with a Zeiss Ultraphot microscope under epifluorescent illumination with wide-band UV and fluorescein filters. The hilus of the dentate gyrus contralateral to the injection site was examined for GADpositive immunoreactive neurons, which were retrogradely labeled with the fluorescent tracer. Double-labeled neurons were identified by switching the filters back and forth. Thus, GAD-positive neurons were obscrved with the fluorescein filter and were compared to the commissural neuronal somata that were observed with the wide-band UV filter.

The sections from brains injected with WGA-HRP were processed according to the technique described by Wainer and Rye (1984) using tetramethylbenzidine (TMB) as the chromogen (Mesulam, 1982). The TMB reaction was stabilized in diaminobenzidine (DAB) and the sections were then processed for GAD immunocytochemistry (Peterson et al., 1985) using an avidin-biotin technique (Hsu et al., 1981) and DAB as the chromogen. These sections were mounted onto gelatin-coated slides, dried, cleared, and covered using DPX mounting medium. The hilus of the dentate gyrus contralateral to the injection site was examined under bright-field illumination for GAD-immunoreactive neurons that were colocalized with retrogradely transported HRP granules.

\section{Anterograde degeneration of the commissural pathway}

Five adult albino rats were anesthetized with chloral hydrate and placed into a stereotaxic head holder. After a large hole was drilled in the cranium, the dorsal hippocampus was removed by aspiration under visual guidance, following the removal of overlying cerebral cortex. After $48 \mathrm{hr}$, the animals were killed and the brains were fixed using the same method described below for the anterograde HRP transport study. The extent of the hippocampal ablations was determined in $100-\mu \mathrm{m}$ thick Vibratome sections. Specimens were obtained from brains in which the dorsal hippocampus was completely ablated and the fornix remained intact. Specimens for electron microscopy were obtained from the hemisphere contralateral to the lesions and processed in a routine manner for thin-sectioning on an ultramicrotome. Ultrathin sections from these specimens were stained with uranyl acetate and lead citrate before examination with the electron microscope.

\section{Anterograde transport of HRP to label the commissural pathway}

Four adult Sprague-Dawley albino rats were anesthetized with $35 \%$ chloral hydrate $(0.1 \mathrm{mg} / 100 \mathrm{gm}$ body weight, i.p.) and injected along with $0.1-0.2 \mu 130 \%$ HRP in distilled water into the dentate gyrus, using stereotaxic guidance. Injections were made in $0.25 \mu 1$ increments and were completed in 10-20 min. Two days later, the rats were again anesthetized with chloral hydrate and fixed by intracardiac perfusion similar to that described above, except that the fixative contained $4 \%$ paraformaldehyde and $1 \%$ glutaraldehyde in $0.12 \mathrm{M}$ phosphate buffer (pH 7.2). These perfused rats were refrigerated overnight at $4^{\circ} \mathrm{C}$, and the brains removed from the cranium the following day.

Brains were sectioned on a Vibratome at a thickness of $100 \mu \mathrm{m}$ and those sections that contained the hippocampus were processed to reveal the location of HRP by incubating them in a saturated solution of diaminobenzidine and $0.01 \% \mathrm{H}_{2} \mathrm{O}_{2}$. After two $10 \mathrm{~min}$ washes, sections were placed onto slides and examined. Those sections that had injection sites localized to the middle part of the dorsal hippocampus were used for electron-microscopic analysis. Small blocks of tissue that contained the dentate gyrus contralateral to the injection site were postfixed in $2 \%$ $\mathrm{OsO}_{4}$, dehydrated in ethanol, and embedded in Epon. Ultrathin sections obtained from these blocks were examined in the electron microscope.

\section{Results}

\section{Double-labeling studies}

Injections of Fast blue, Fluoro-Gold, or WGA-HRP were centered in the septal third of the hilus of the dentate gyrus and partly in CA3/c. In the contralateral hippocampal formation, numerous retrogradely labeled neurons were found in the CA3 and hilus (Figs. 1, 3, and 5). The shapes, sizes and distribution of these neurons were consistent with previously published results.

Sections processed for GAD immunocytochemistry were obtained from brains in which the injection sites were localized to the hilus and where retrograde transport had been confirmed. Several neurons of different shapes and sizes in the hilus showed double-labeling for one of the retrograde markers and GAD (Figs. 1-6). These double-labeled cells were found both contralateral and ipsilateral to the injection sites. One of the more frequent types of double-labeled neuron had a bipolar shape and was located near the granule cell layer (Figs. 3 and 4). Others were located deeper in the hilus and had round somata. Usually, only 1-2 neuronal somata per section displayed the doublelabeling. A quantitative analysis was made from 5 brains in which the injection sites were well placed and the immunocytochemical staining was excellent. Approximately 15 sections from each brain were analyzed. In total, over 2000 retrogradely labeled neurons were observed, and 110 of these cells were double-labeled. This finding indicates that approximately $5 \%$ of the total number of retrogradely labeled neurons in the hilus are GABAergic.

In the same cases, double-labeled cells were infrequently observed in the pyramidal cell layer of CA3 (Figs. 5 and 6). They had large, round somata and were adjacent to unlabeled pyramidal cells.

\section{Anterograde studies}

In the experiments in which the dorsal hippocampus was lesioned, degenerating terminals were found in various layers of the contralateral hippocampal formation. They were most numerous in stratum oriens of CAl (Figs. 7-9) and less dense in stratum radiatum. Degenerating terminals were also present in these 2 layers in CA3 and in the dentate gyrus, where the majority were observed in the molecular layer (Figs. 10 and 11), although others were found in the hilus and granule cell layer. They formed mainly asymmetric synapses with spines and dendritic shafts (Fig. 7). However, a small number of degenerating terminals formed symmetric synapses (Figs. 8-11). These latter terminals formed synapses with variously sized dendritic shafts and spines.

The preparations in which HRP was used to label the commissural axons provided similar results. The distribution of labeled commissural terminals in the hippocampal formation was the same as that for the degenerating commissural terminals. Most of the terminals that contained the anterogradely 

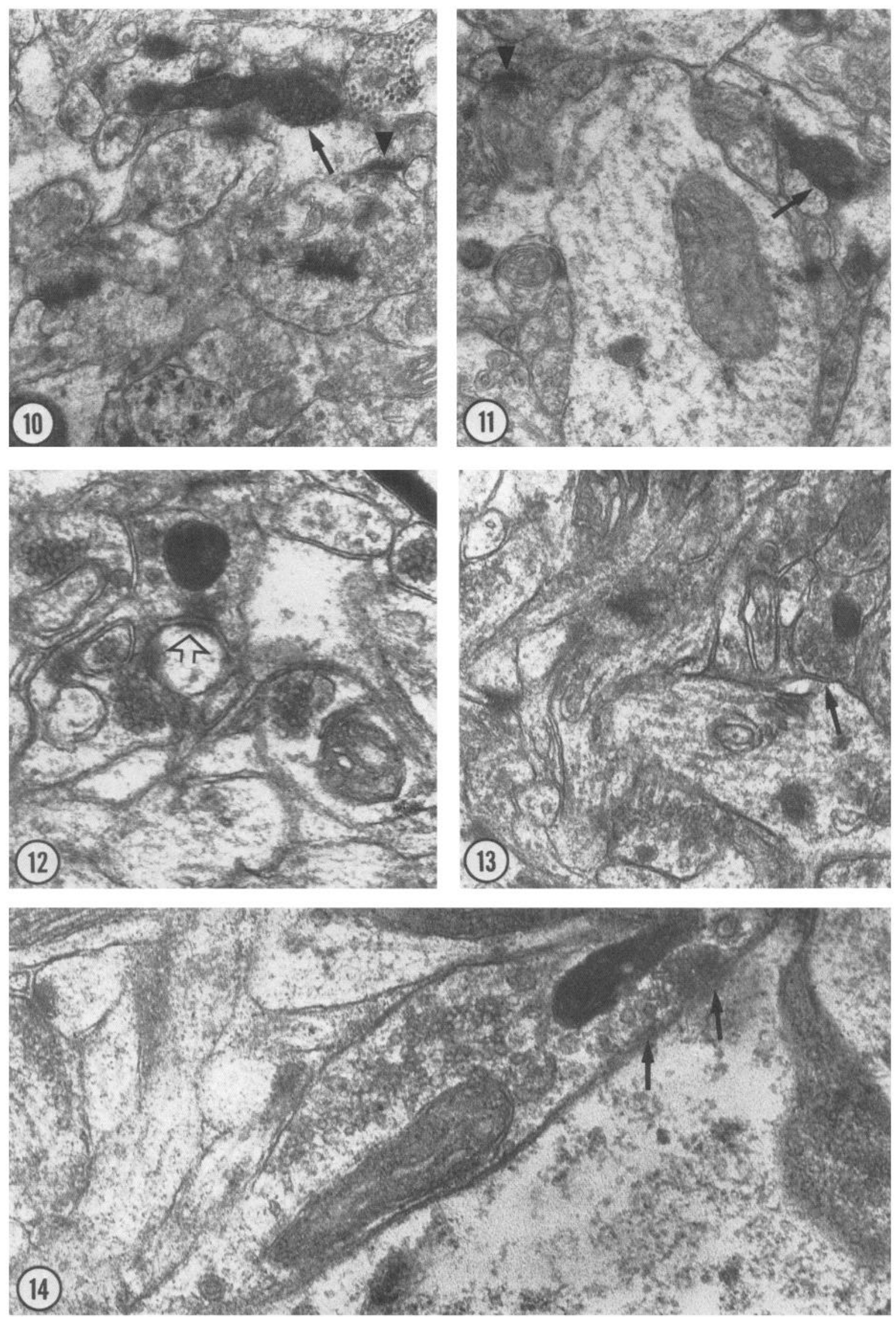
transported HRP reaction product formed asymmetric synapses (Fig. 12). However, a small number of terminals that formed symmetric synapses could also be found in CA1 and in the lower molecular layer of the dentate gyrus (Figs. 13 and 14). This latter population of terminals formed about $5 \%$ of the total number of HRP-labeled terminals in the dentate gyrus.

\section{Discussion}

The commissural pathway of the hippocampal formation has been studied extensively in the last 2 decades. Most of these studies have concluded that this pathway is exclusively excitatory (Deadwyler et al., 1975). However, in one of the electron microscopic descriptions, Laatsch and Cowan (1967) raised some doubt about this notion in that they observed some symmetric contacts in the dentate gyrus formed by degenerating commissural axons. Our previous findings showed large numbers of GABAergic hilar neurons (Ribak et al., 1978; Seress and Ribak, 1983) and GABAergic-like ultrastructural features for some hilar commissural neurons (Seroogy et al., 1983), and these data suggested that some GABAergic hilar neurons had axons that projected through the hippocampal commissure. The present double-labeling data conclusively demonstrate this relationship for the hilus-to-hilus connection (Figs. 1-4) and the CA3-CA3 connection (Figs. 5 and 6). The anterograde degeneration and HRP transport data of terminals that form symmetric synapses are consistent with this notion of GABAergic commissural connections for these 2 regions, as well as for the CA3-to-CA1 commissural connection (Figs. 7-9). Therefore, these data are the first direct demonstration of a GABAergic projection neuron in the cerebral cortex. Further studies are required to determine whether this finding is restricted to allocortex or if neocortex displays some GABAergic projection neurons.

Other data are consistent with the notion that cortical GABAergic terminals are not derived exclusively from local circuit neurons. For example, Köhler et al. (1984) have demonstrated the existence of GABAergic cells that have projections to the hippocampus in the medial septum and the diagonal band of Broca. In addition, Vincent et al. (1983) have identified, in the posterior hypothalamus, GABAergic neurons that project to diverse cortical regions. Axons from these 2 GABAergic sources were not interrupted in this study because the electronmicroscopic analysis utilized hemispheres that had an intact ipsilateral fornix. These data, as well as the present results, contradict the biochemical data that show no significant reduction in GABA markers after surgical isolation of either the hippocampus or cerebral cortex (Emson and Lindvall, 1979; Fonnum and Walaas, 1978). One proposed explanation that would take into account all of these data suggests that most cortical GABA arises from intrinsic neurons and that these 3 extrinsic GABAergic pathways (septal-hippocampal, commissural, and hypothalamo-cortical) represent relatively small inputs.

The commissural pathway was thought to terminate on dentate gyrus granule cells as an exclusively excitatory input. $\mathrm{Re}-$ cently, it has been shown that commissural terminals contact not only the granule cells but also the basket cells in the dentate gyrus (Frotscher and Zimmer, 1983; Seress and Ribak, 1984). Since basket cells provide inhibitory input to granule cells, this finding supports the electrophysiological observations that feedforward inhibition occurs via the commissural pathway (Buzsáki and Eidelberg, 1981). In these electrophysiological exper- iments, however, the appearance of the inhibition has a latency that lies between the mono- and disynaptic period, and this suggests that the inhibition can be caused directly by monosynaptic inhibitory axons through the commissural pathway to the granule cells. The present findings of double-labeled, GAD-positive commissural neurons in the hilus as well as degenerating and HRP-labeled terminals that form symmetric synapses in the molecular layer are consistent with this notion of direct inhibition in the commissural pathway.

Our earlier data on the number of GABAergic hilar neurons (Seress and Ribak, 1983) strongly suggested that an inhibitory component existed in the commissural pathway to the contralateral dentate gyrus. The double-labeling experiments have clearly demonstrated that a small proportion of these GABAergic hilar neurons have commissural projections. This GABAergic component of the commissural pathway seems to be smaller than expected in light of the observation that more than half of the hilar cells are GABAergic. However, the results might not reflect the true size of this inhibitory projection because (1) the entire hilar region from which these projections arise was not filled with the retrograde tracers or (2) the branching pattern of individual commissural axons from GABAergic cells is not very large compared to their local axons, so that less tracer is retrogradely transported.

Although our interest in a GABAergic component of the commissural pathway started with the dentate gyrus, it is apparent from the double-labeling results that this component also exists for the CA3-CA3 commissural connection. The data on the commissural axon terminals in CAl also suggest that a GABAergic component may occur in this part of the hippocampal commissural pathway. Double-labeling studies are needed to determine whether the source of this pathway is GAD-positive neurons in the CA3 pyramidal cell layer or those in strata radiatum and oriens.

In conclusion, our data indicate that the hippocampal commissural pathway includes a minor GABAergic inhibitory component. Thus, direct inhibition, as well as feed-forward inhibition, may exist in the commissural pathway of the rat hippocampus.

\section{References}

Alger, B. E., and R. A. Nicoll (1982) Pharmacological evidence for two kinds of GABA receptor on rat hippocampal pyramidal cells studied in vitro. J. Physiol. (Lond.) 328: 125-141.

Berger, W. B., S. Semple-Rowland, and J. L. Bassett (1981) Hippocampal polymorph neurons are the cells of origin for ipsilateral association and commissural afferents to the dentate gyrus. Brain Res. 215: 329-336.

Buzsáki, G., and E. Eidelberg (1981) Commissural projection to the dentate gyrus of the rat: Evidence for feed-forward inhibition. Brain Res. 230: 346-350.

Coons, A. H. (1958) Fluorescent antibody methods. In General Cytochemical Methods, J. F. Danielli, ed., pp. 399-422, Academic, New York.

Curtis, D. R., D. Felix, and H. McLennan (1970) GABA and hippocampal inhibition. Br. J. Pharmacol. 40: 881-883.

Deadwyler, S. A., J. R. West, C. W. Cotman, and G. S. Lynch (1975) A neurophysiological analysis of commissural projections to dentate gyrus of the rat. J. Neurophysiol. 38: 167-184.

Emson, P. C., and O. Lindvall (1979) Distribution of putative neurotransmitters in the neocortex. Neuroscience 4: $1-42$.

Fonnum, F., and I. Walaas (1978) The effect of intrahippocampal

\footnotetext{
Figures 10-14. Figures 10 and 11 give examples of degenerating terminals that form symmetric synapses (arrows) in the inner third of the molecular layer of the dentate gyrus. $\times 15,600$ and $\times 31,100$, respectively. Figures $12-14$ show electron micrographs of HRP-labeled axon terminals in the molecular layer of the dentate gyrus contralateral to the injection site. Figure 12 shows a terminal with a large HRP granule that forms an asymmetric synapse (open arrow) with a spine. $\times 37,300$. Figures 13 and 14 show examples of HRP-labeled terminals with pleomorphic vesicles that form symmetric synapses (arrows) with dendrites. $\times 40,000$ and $\times 41,600$, respectively.
} 
kainic acid injections and surgical lesions on neurotransmitters in hippocampus and septum. J. Neurochem. 31: 1173-1181.

Frotscher, M., and J. Zimmer (1983) Commissural fibers terminate on non-pyramidal neurons in the guinea pig hippocampus-a combined Golgi-EM degeneration study. Brain Res. 265: 289-293.

Hsu, S.-M., I. Raine, and H. Fanger (1981) Use of avidin-biotinperoxidase complex $(\mathrm{ABC})$ in immunoperoxidase techniques: A comparison between $\mathrm{ABC}$ and unlabeled antibody (PAP) procedures. J. Histochem. Cytochem. 29: 577-580.

Köhler, C., V. Chan-Palay, and J. -Y. Wu (1984) Septal neurons containing glutamic acid decarboxylase immunoreactivity project to the hippocampal region in the rat brain. Anat. Embryol. 169: 41-44.

Kosaka, T., K. Hama, and J.-Y. Wu (1984) GABAergic synaptic boutons in the granule cell layer of rat dentate gyrus. Brain Res. 293. 353-359.

Krnjević, K. (1974) Chemical nature of synaptic transmission in vertebrates. Physiol. Rev. 54: 418-540.

Laatsch, R. H., and W. M. Cowan (1967) Electron microscopic studies of the dentate gyrus of the rat. II. Degeneration of commissural afferents. J. Comp. Neurol. 130: 241-262.

Laurberg, S. (1979) Commissural and intrinsic connections of the rat hippocampus. J. Comp. Neurol. 184: 685-708.

Mesulam, M.-M. (1982) Tracing Neuronal Connections with Horseradish Peroxidase, Wiley, Chichester, UK.

Oertel, W. H., D. E. Schmechel, E. Mugnaini, M. L. Tappaz, and I. J. Kopin (1981) Immunocytochemical localization of glutamate decarboxylase in rat cerebellum with a new antiserum. Neuroscience 6 : 2715-2735.

Peterson, G. M., C. E. Ribak, and W. H. Oertel (1985) A regional increase in the number of hippocampal GABAergic neurons and terminals in the seizure-sensitive gerbil. Brain Res. 340: 384-389.

Ribak, C. E., J. E. Vaughn, and K. Saito (1978) Immunocytochemical localization of glutamic acid decarboxylase in neuronal somata following colchicine inhibition of axonal transport. Brain Res. 140:315332.

Ribak, C. E., L. Seress, K. B. Seroogy, G. M. Peterson, and W. H. Oertel (1985) The hippocampal commissural pathway contains a GABAergic inhibitory component. Anat. Rec. 211: 160A.
Schmued, L. C., and J. H. Fallon (1986) Fluoro-Gold: A new fluorescent retrograde axonal tracer with numerous unique properties. Brain Res. 377: 147-154.

Seress, L., and C. E. Ribak (1983) GABAcrgic cells in the dentate gyrus appear to be local circuit and projection neurons. Exp. Brain Res. 50: 173-182.

Seress, L., and C. E. Ribak (1984) Direct commissural connections to the basket cells of the hippocampal dentate gyrus. Anatomical evidence for feed-forward inhibition. J. Neurocytol. 13: 215-225.

Seroogy, K. B., L. Seress, and C. E. Ribak (1983) Ultrastructure of commissural neurons of the hilar region in the hippocampal dentate gyrus. Exp. Neurol. 82: 594-608.

Somogyi, P., A. D. Smith, M. G. Nunzi, A. Gorio, H. Takagi, and J.Y. Wu (1983) Glutamate decarboxylase immunoreactivity in the hippocampus of the cat. Distribution of immunoreactive synaptic terminals with special reference to the axon initial segment of pyramidal neurons. Neuroscience 3: 1450-1468.

Swanson, L. W., J. M. Wyss, and W. M. Cowan (1978) An autoradiographic study of the organization of intrahippocampal association pathways in the rat. J. Comp. Neurol. 181: 681-716.

Swanson, L. W., P. E. Sawchenko, and W. M. Cowan (1981) Evidence for collateral projections by neurons in Ammon's horn, the dentate gyrus and the subiculum: A multiple retrograde labeling study in the rat. J. Neurosci. 1: 548-559.

Vincent, S. R., T. Hökfelt, L. R. Skirboll, and J.-Y. Wu (1983) Hypothalamic $\gamma$-aminobutyric acid neurons project to the neocortex. Science 220: 1309-1311.

Voneida, T. J., R. M. Vardaris, S. E. Fish, and C. T. Reiheld (1981) The origin of the hippocampal commissure in the rat. Anat. Rec. 201: 91-103.

Wainer, B. H., and D. B. Rye (1984) Retrograde horseradish peroxidase tracing combined with the localization of cholinc-acctyltransferase immunoreactivity. J. Histochem. Cytochem. 32: 439-443.

West, J. R., H. O. Nornes, C. L. Barnes, and M. Bronfenbrenner (1979) The cells of origin of the commissural afferents to the area dentata in the mouse. Brain Res. 160: 203-215. 